\title{
Social Network Platforms in Educational Settings: A Network Analysis Ap- proach to Analyze Online Student Interactions
}

\section{Prof. Tzu-Liang Bill Tseng, University of Texas, El Paso}

Dr. Tseng is a Professor and Chair of Industrial, Manufacturing and Systems Engineering at UTEP. His research focuses on the computational intelligence, data mining, bio- informatics and advanced manufacturing. Dr. Tseng published in many refereed journals such as IEEE Transactions, IIE Transaction, Journal of Manufacturing Systems and others. He has been serving as a principle investigator of many research projects, funded by NSF, NASA, DoEd, KSEF and LMC. He is currently serving as an editor of Journal of Computer Standards \& Interfaces.

\section{Aditya Akundi, University of Texas, El Paso}

Aditya Akundi is currently a doctoral student at the University of Texas at El Paso in the Electrical and Computer Engineering Department, Industrial and Systems Engineering (ISE) track. He earned a Master of Science in Electrical and Computer Engineering at the University of Texas at El Paso (UTEP) in 2012. $\mathrm{He}$ has worked on a number of projects in the field of Electrical \& Computer Engineering, Systems Engineering, Additive Manufacturing and Green Energy Manufacturing. He is the current president of INCOSE UTEP student chapter along with being involved in UTEP Green Fund committee. His research interests are in Systems Engineering \& Architecture, Complex systems, Systems testing and Application of Entropy to Complex Systems. Email: sakundi@ @iners.utep.edu

\section{Dr. Richard Chiou, Drexel University}

Dr. Richard Chiou is Associate Professor within the Engineering Technology Department at Drexel University, Philadelphia, USA. He received his Ph.D. degree in the G.W. Woodruff School of Mechanical Engineering at Georgia Institute of Technology. His educational background is in manufacturing with an emphasis on mechatronics. In addition to his many years of industrial experience, he has taught many different engineering and technology courses at undergraduate and graduate levels. His tremendous research experience in manufacturing includes environmentally conscious manufacturing, Internet based robotics, and Web based quality. In the past years, he has been involved in sustainable manufacturing for maximizing energy and material recovery while minimizing environmental impact. 


\title{
Social Network Platforms in Educational Settings: A Network Analysis Approach to Analyze Online Student Interactions
}

\begin{abstract}
Interactions create webs of interconnected activities, and may give rise to emergent phenomenon. The word interaction in educational settings refers to person-to-person communication, and thus exists in in-class discussions, in-class debates, and so forth. Educational settings that emerge from such collaborations give rise to a network of interactions among the stakeholders involved. This paper briefs an exploration on the concepts of social network analysis and their ability to shed light on the impacts of incorporating social network platforms in educational settings. In this paper we try to analyze the information flow based on undergraduate and graduate student online interaction patterns. An online Facebook group was created as a part of this study to initiate a computer mediated communication platform for the geographically separated students to connect and engage in classroom based meaningful discussions. We use Netvizz, a data extraction tool to collect data from student groups in Facebook social networking platform. The networks constructed using the data gathered at five random time steps are analyzed using individual node degrees both qualitatively and quantitatively for understanding the interaction patterns observed.
\end{abstract}

\section{Introduction}

The word interaction is defined to be a phenomenon where two or more objects have an effect upon each other. Several such interactions when merged together give rise to a set of interconnected and interdependent activities. Interactions in educational settings can be referred to the stakeholders of the whole academic enterprise connected with each other towards a goal of continual learning and innovation. In-Class student interactions, Teacher-Student interactions, Inclass subject based discussions and several similar scenarios that come to play in educational settings can be referred to a phenomenon having effect upon each other in academic settings.

The concept, Interaction hypothesis, proposed by Second Language Acquisition expert (SLA) Michael Long ${ }^{1}$ elaborates of a way in which ESOL (English for speakers of other languages) students can learn a target language by conversation. This implies that interactions help towards developing language proficiency in ESOL students. Similarly, in educational settings, active academic collaboration among professors, peers and students is gaining more importance and attention $^{2}$. Collection of such several such collaborations emerge to form a network among the stakeholders involved. Organizing and identifying these interactions will help towards strategically well-developed classroom settings. Flipped classroom falls under the umbrella of extending the learning boundaries based on interactive students centered techniques. Many educational institutions are currently embracing the technique of flipped classrooms. According to Lage et al, inverted (flipped) classroom can be defined as a setting where the events that usually take place inside a classroom in a traditional setting, now take place outside the classroom. An example the authors use here to describe inverted classroom is the use of World Wide Web and multimedia communications as an opportunity for students to view lectures ${ }^{3}$. Bishop et al., define a flipped classroom as an educational setting that promotes: (a) Interactive group learning activities inside the classroom (b) Computer based individual instruction outside the classroom. With this observed increase in the use of technology for effectively engineering 
student educational experience, several new tools and platforms are currently being developed and explored ${ }^{4}$.

The authors of this paper are currently working towards exploring effective pedagogical methods that promote student learning and student interactions. As a part of this effort, to understand social networks based student interactions for educational settings, an online secure Facebook group was developed to encourage students from The University of Texas at El Paso (UTEP) and Drexel University (DU) to interact and collaborate on the topics of Green Energy and Green Manufacturing. The interaction patterns of undergraduate and graduate students enrolled in Green Energy and Manufacturing classes at UTEP and DU are extracted and studied by the authors using network analysis tools and the concepts from information theory to analyze the flow of information among students using social network tools geared towards learning for education settings.

\section{Social Network Platform \& Data Gathering}

Social Networks emerge from a patterned arrangement of interactions based on the actions by individuals in a society. From a hierarchical perspective, it portrays a network of ties among a set of individuals at a lower level and at a higher level it represents ties and patterns of emergence in a social structures. Two broader divisions of social networks fall into "ego centric" and "socio centric" networks where; ego centric networks portray an individual actor and the effect of the network on that individual whereas, social centric network portray a set of individuals and their patterns of interactions ${ }^{5}$. Facebook is a freely available one of the most used social networking tool among students. Taking into consideration its user friendly interface to connect and share information among a set of actors, this social networking platform was used to understand information flow in education centric student interaction patterns.

A private Facebook group for the students of Engineering Technology Dept. from DU and the students of Industrial, Manufacturing and Systems Engineering Dept. at UTEP enrolled for Green Energy manufacturing class was created. Undergraduate and Graduate students with a background in Industrial Engineering, Manufacturing Engineering, Systems Engineering and Engineering Technology from both universities were given access to this group. Creation of this group was aimed at providing collaborative interactive sessions among the enrolled geographically separated students to participate in class related discussions, sharing freely available resources, and proving individual student insights in the topics and trends related to Green Energy \& Green Manufacturing. Students with access to the Facebook group were encouraged to post their insights on emerging green energy technologies and share their in-class and project based experience. The class instructor acting as group moderator posted discussion boards weekly based on student curriculum progress, which enabled students to discuss on their individual insights weekly based on the posts. The class instructor's act as effective moderators in educational setting based social network groups. This is because we believe that the instructor has a better insight on the weekly progress of students and their understanding of the subject based on which appropriate discussion boards can be initiated. Discussion on student class projects were also encouraged and observed in this group ${ }^{11}$. Interactions of the enrolled students in the Facebook group over the semester emerged into a student centric academic interaction network. 
This network consists of a set of actors (23 students in total who joined the group created), in this case enrolled students from both universities linked/connected by a relationship observed. It is important to note that all the course enrolled students at both universities were encouraged to join the group however; it was observed that only 23 participated out of 35 students.

As a first step towards analyzing student interactions, some of the topics discussed upon in the group were $^{11}$ : (a) Benefits of implementing Green energy systems; (b) Students perception of what a Green energy systems is and potential of green energy system implementation; (c) Benefits of wind energy and implications of Wind energy Systems; (d) Benefits of solar energy and implications of Solar energy systems; (e) Benefits of Green Energy and Green Energy Manufacturing.

In order to map a network at the conclusion of the semester based on the discussions, posts, and comments observed in the group by both the students and moderators, Netvizz ${ }^{\circledR}$ a data extraction tool was used to extract data from Facebook group. This data was then imported into Gephi® software, an interactive network visualization platform. Figure 1 illustrates the raw network extracted from the Facebook group. To better visualize, understand and see interactions in the network, social network analysis tools available in Gephi were used which resulted in the network illustrated in figure 2 .

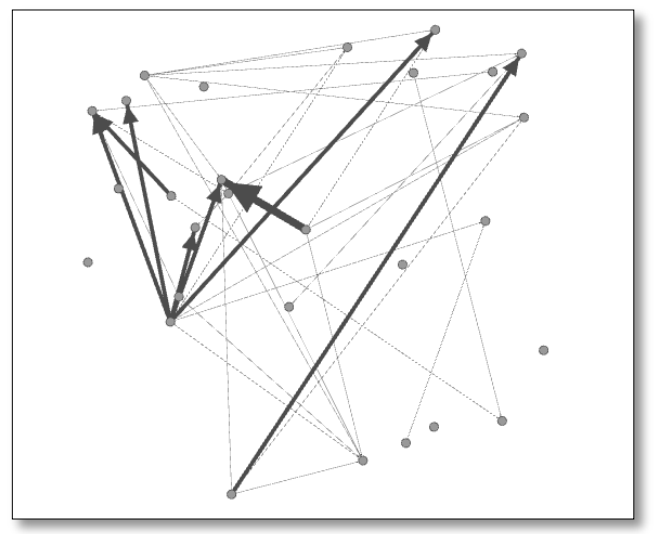

Figure 1. Raw extracted network based on student interaction in the Facebook group

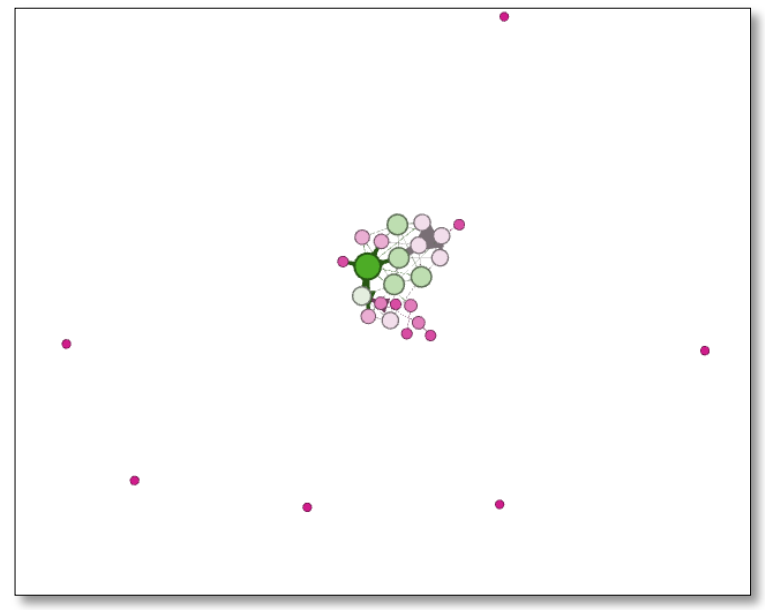

Figure 2. Visualization of the network from figure 1 
The nodes illustrated in the figure represent the actors (students) of the network and the edges i.e. the connections between the students (nodes) represent that a given node commented, liked or shared the post made by the node at its corresponding edge (connection). The thickness of the edges represent the number of interactions among the nodes, the thicker the edge more often the nodes interacted with each other. From figure 2, it can be seen that there are outliers in the network i.e. not all students registered in the Facebook group were actively participating in the discussions. To further understand the student interaction behavior, the outliers observed in figure 2 are omitted from the analysis thereby resulting in the network illustrated in figure 3.



Figure 3. Illustration of student group interactions after omitting the outliers

\section{Information Theory}

This study aimed to understand the information flow patterns in the network; concepts from information theory were used to quantify the information flow among the nodes. Information can broadly be defined as a set of data that is either organized for a specific purpose, or presented within a specific context, giving it meaning and relevance ${ }^{6}$. In the context of this paper, information can be defined as knowledge transferred among the participants of the created Facebook group. This transfer of knowledge can be looked at as student gaining new knowledge based on the student he/she interacts with, leading to an increased understanding of the content of a course.

Information theory is a branch of mathematics based on the theory of probability and statistics. It is applied in various fields where probability and statistics play an important role. It is a system of measurement used for quantifying information conveyed by the occurrence of an event ${ }^{9,10}$. This event can be either a response or a stimulus that is a function of a number of possible events and 
their probabilities ${ }^{9,10}$. The concept Entropy, anchored to information theory is used in this paper towards trying to quantify the flow of information among the nodes of the network. Entropy based on information theory is defined as "A measure of uncertainty associated with a random

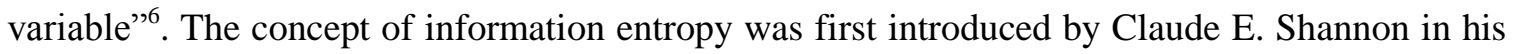
paper “A Mathematical Theory of Communication' in 1948.

Entropy thus represents a measure of information defined by $H(x)=-\sum_{i=1}^{x} P(x) \log P(x)^{7,8}$. This measure enables in the reduction of uncertainty of a measurement $x$ of a random variable with a probability $\mathrm{p}(x)$. To further extend Shannon entropy, towards measuring 2 random variables $\mathrm{X}$ and $\mathrm{Y}$ at different scales, a measure of mutual information is used. However, based on the fact that the measure of mutual information being symmetrical i.e. $I(X, Y)=I(Y, X)$ which implies that the future state of a random variable has a causal effect on the past state. Thereby, in order to address this symmetrical limitation we use the concept of transfer entropy ${ }^{7}$. The concept of Transfer Entropy (TE) was given by Schreiber to address the time symmetric limitation of mutual information measure. For a considered two sample spaces of information, transfer entropy is defined as the additional amount of information gained based on the next observation of one of the two systems being considered. TE, the transfer of information between two random variables $\mathrm{X}$ and $\mathrm{Y}$ is given $\mathrm{by}^{7,8}$ :

$$
T E(X, Y)=\sum_{t=1} p\left(y_{t+1}, y_{t}, x_{t}\right) \log \frac{p\left(y_{t+1}, y_{t}, x_{t}\right) \cdot p\left(y_{t}\right)}{p\left(y_{t}, x_{t}\right) \cdot p\left(y_{t+1}, y_{t}\right)}
$$

Also, the transfer entropy from $\mathrm{Y}$ to $\mathrm{X}$ can be inferred similarly based on the equation portrayed.

\section{Transfer Entropy applied to Student Network}

To analyze the flow of information generated from one node to the other of a considered network based on the observed individual node degree evolution over several time steps of network formation, the concept of transfer entropy (TE) is used. TE when applied to the current scenario helps to understand how much of information generated at one node (student) is responsible for the information obtained by the other node (student). To generate TE values based on individual node degree evolution, data on the number of nodes present along with the individual node degrees over 5 different time steps were captured based only on the student interactions in Facebook group discussions over the semester. Figures 4-8 illustrates the evolution of interactions in the student Facebook group over five different snapshots. It also illustrates on how students start interacting with each other over the semester. To protect student identity, the nodes are marked with numbers.

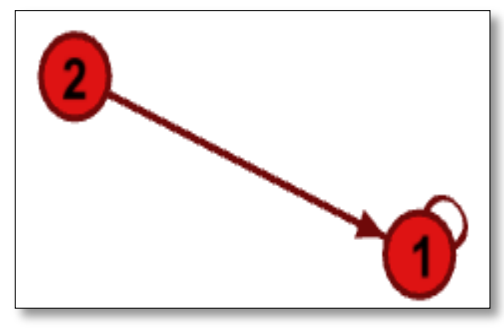

Figure 4. Network observed at time step 1 


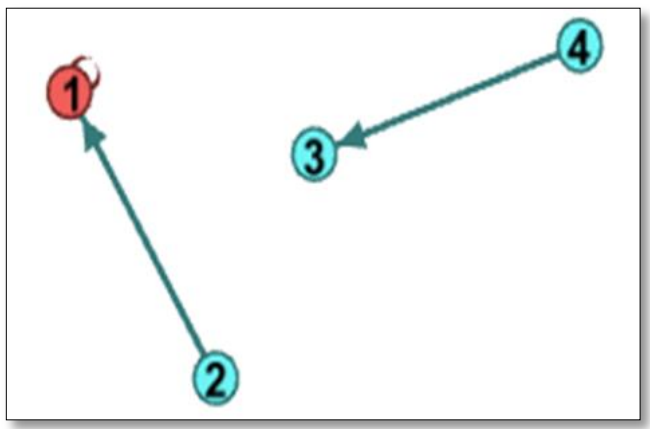

Figure 5. Network observed at time step 2

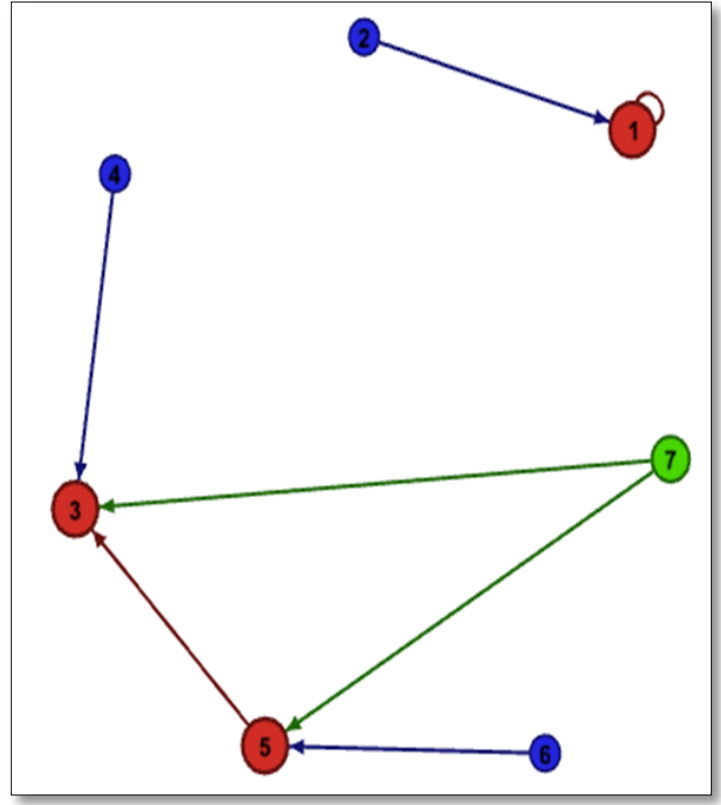

Figure 6. Network observed at time step 3

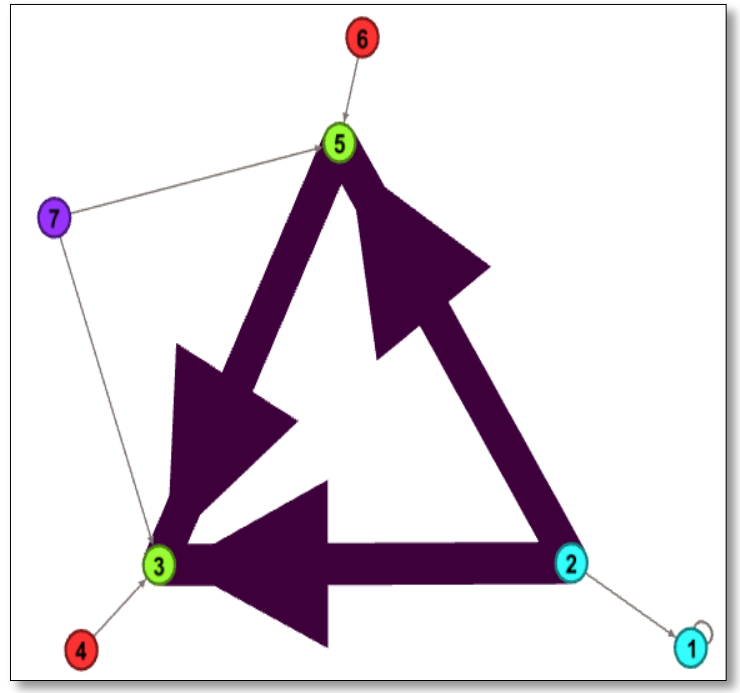

Figure 7. Network observed at time step 4 


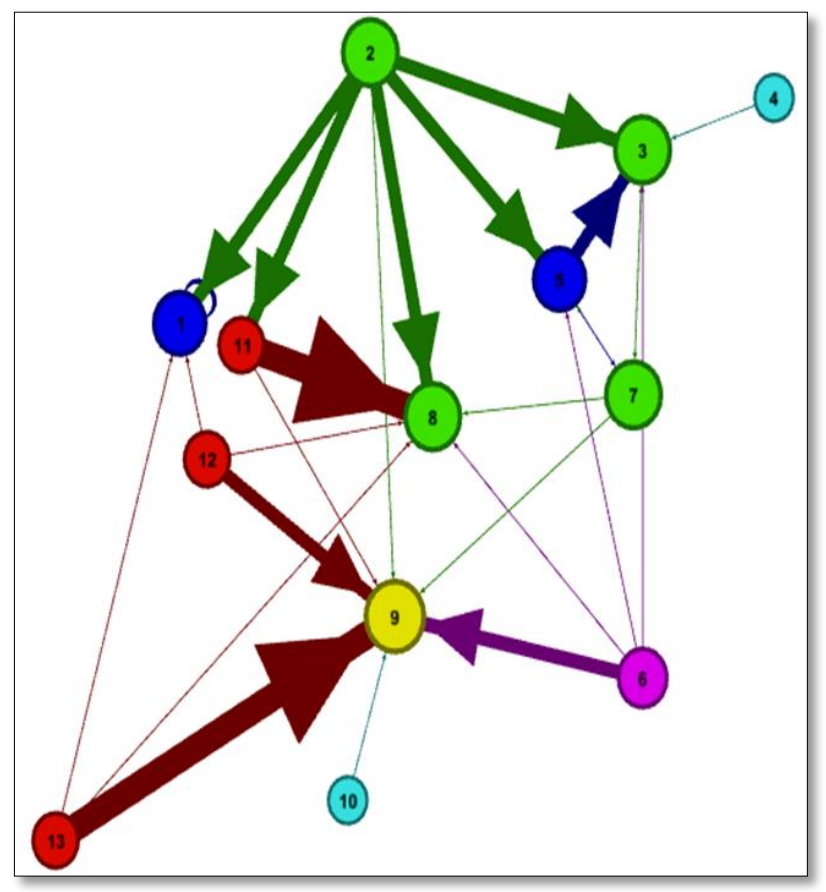

Figure 8. Network observed at time step 5

To read the captured data that is illustrated in table 1 based on the 5 different network snapshots captured and illustrated in figures 4-8, the degree evolution of node 1 over 5 different time steps is represented by the second row of the table, degree evolution of node 2 is represented by the second row of the table and so on.

Table 1. Node degree evolution over the time steps captured. (Note: N1 to N13 represent the different nodes in the network; T1 to T5 represent the time steps)

\begin{tabular}{|c|c|c|c|c|c|}
\hline & T1 & T2 & T3 & T4 & T5 \\
\hline N1 & 3 & 3 & 3 & 3 & 5 \\
\hline N2 & 1 & 1 & 1 & 3 & 6 \\
\hline N3 & 0 & 1 & 3 & 4 & 6 \\
\hline N4 & 0 & 1 & 1 & 1 & 1 \\
\hline N5 & 0 & 0 & 3 & 4 & 5 \\
\hline N6 & 0 & 0 & 1 & 1 & 4 \\
\hline N7 & 0 & 0 & 2 & 2 & 6 \\
\hline N8 & 0 & 0 & 0 & 0 & 6 \\
\hline N9 & 0 & 0 & 0 & 0 & 7 \\
\hline N10 & 0 & 0 & 0 & 0 & 1 \\
\hline $\mathbf{N 1 1}$ & 0 & 0 & 0 & 0 & 3 \\
\hline $\mathbf{N 1 2}$ & 0 & 0 & 0 & 0 & 3 \\
\hline $\mathbf{N 1 3}$ & 0 & 0 & 0 & 0 & 3 \\
\hline
\end{tabular}

For calculating the TE values represented in table 2, based on TE equation, joint probabilities are calculated for emerging node degrees observed in table 1. 
Table 2. Transfer Entropy values calculated based on table 1

\begin{tabular}{|c|c|c|c|}
\hline $\begin{array}{c}\text { Source Node } \\
\text { (S) }\end{array}$ & $\begin{array}{c}\text { Destination Node } \\
\text { (D) }\end{array}$ & $\begin{array}{c}\text { Transfer Entropy } \\
\text { (S-D) }\end{array}$ & $\begin{array}{c}\text { Transfer Entropy } \\
\text { (D-S) }\end{array}$ \\
\hline N1 & N2 & 0 & 0.2442191 \\
\hline N2 & N3 & 0 & 0.2073259 \\
\hline N3 & N4 & 0.09370405 & 0 \\
\hline N4 & N5 & 0.150515 & 0.09370405 \\
\hline N5 & N6 & 0.150515 & 0 \\
\hline N6 & N7 & 0 & 0 \\
\hline N7 & N8 & 0.09370405 & 0 \\
\hline N8 & N9 & 0 & 0 \\
\hline N9 & N10 & 0 & 0 \\
\hline N10 & N11 & 0 & 0 \\
\hline N11 & N12 & 0 & 0 \\
\hline N12 & N13 & 0 & 0 \\
\hline
\end{tabular}

\section{Discussion}

There are several existing network analysis methods such as finding: Centrality measures to understand the importance of each actor (node) and their influence on the network; Bridges to identify actors (nodes) that act as ties in a network, Degree of Separation to identify the number of ties to connect two actors; Homophily to understand how actors (nodes) tie to similar and dissimilar nodes and so on. All the said measures quantify the structural aspects of a network at a given snapshot. Using the concept of transfer entropy, here we try to understand the dynamicity of the network by considering network evolution over a given time period. Analyzing the graph illustrated in figure 9, based on table 2; TE values suggest the flow of information from a given node to the other. In the graph (Figure 9), the $\mathrm{X}$ axis represents the node numbers and $\mathrm{Y}$ axis represents the corresponding TE values. It is read as: for the red line, label 1 is the TE value from Node 1 to Node 2; label 2 is the TE value from Node 2 to Node 3 and so on. For the blue line, label 1 is the TE value from Node 2 to Node 1, label 2 is the TE value from Node 3 to Node 2 and so on.

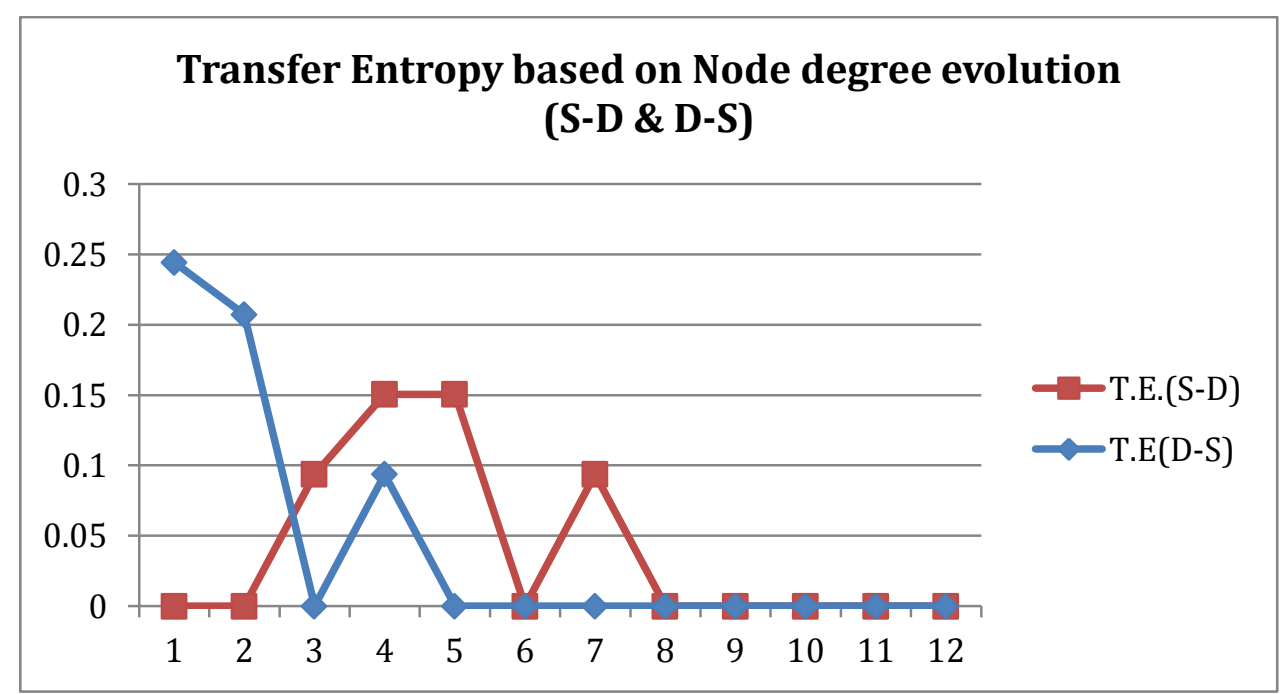

Figure 9. Transfer entropy values between the nodes 
At early stages of the graph, the red line when observed, the value of TE from node 1 to node 2 is zero and TE from node 2 to node 3 is zero. This simply suggests the initial network structural formation where, at the first time step, node 2 is connected to node 1 and at the second time step two sub networks are observed with a disconnect between nodes 1,2 and nodes 3,4. Holistically when the whole network is observed, the graph indicates that while the network starts to evolve, the flow of information from one node to the other increases and then decreases to stabilize. This may imply that the students are active initially by posting and sharing information in the group. Once the information is reached to all the group members, the transfer of information among the students slowly decreases. Towards gaining more insight on the flow of information at a lower level from each and every node of the network to the other, transfer entropy values were calculated from each node across the whole network. The results obtained signify that, even if there is more than one interaction between two given nodes it doesn't necessarily represent high information flow between them.

\section{Conclusion}

Aimed at understanding the effectiveness in the use of social networks among students for educational settings, this study illustrates an approach on the use of the concepts from information theory towards quantifying the flow of information from one node of a network to the other based on their degree evolution. Based on the limited data used to map a network for this study, it shows that even if there is more than one interaction observed among two given nodes, it doesn't necessarily signify information flow among them. However, the Facebook group provided a very effective platform for students to discuss and observe the topics related to the trends in green energy and green manufacturing. All the students involved in the group actively participated with an exception of few outliers observed in the network. There are several other network characteristics that can be explored, however to limit the scope of this study the analysis was based only on considering the node degrees of the network. Authors believe the use of Facebook groups as an informal medium in educational setting will prove effective as it provides students with a collaborative environment that engages its participants with constant updates and provides them with different perspectives of their peers towards a same problem. To further use this approach in better understanding and quantifying the flow of information among the students, the authors are currently looking to expand this work to be applied in large scale educational setting based networks. The future direction of the authors include understanding if a given students grade can be correlated with the frequency of interactions in the classroom, and trying to analyze if dissemination of knowledge and information to student network leaders in a given classroom setting will prove effective.

\section{References:}

1. Ellis, Rod. "The Interaction Hypothesis: A Critical Evaluation." (1991)

2. Kang, Changhui. "Academic interactions among classroom peers: a cross-country comparison using TIMSS." Applied Economics 39.12 (2007): 1531-1544.

3. Lage, Maureen J., Glenn J. Platt, and Michael Treglia. "Inverting the classroom: A gateway to creating an inclusive learning environment." The Journal of Economic Education 31.1 (2000): 3043

4. Bishop, Jacob Lowell, and Matthew A. Verleger. "The flipped classroom: A survey of the research." ASEE National Conference Proceedings, Atlanta, GA. 2013. 
5. Carrington, Peter J., John Scott, and Stanley Wasserman, eds. Models and methods in social network analysis. Vol. 28. Cambridge university press, 2005.

6. Cover, Thomas M., and Joy A. Thomas. Elements of information theory. John Wiley \& Sons, 2012.

7. Murcio, Roberto, et al. "Urban transfer entropy across scales." arXiv preprint arXiv:1505.02761 (2015).

8. Schreiber, Thomas. "Measuring information transfer." Physical review letters85.2 (2000): 461.

9. Kullback, Solomon. Information theory and statistics. Courier Corporation, 1997.

10. Proctor, Robert W., and Trisha Van Zandt. Human factors in simple and complex systems. CRC press, 2008.

11. Ruane, Regina, Richard Chiou, and Tseng Bill. "Creating an Online Community to engage and support undergraduate engineering students in Green Energy Manufacturing." Society for Information Technology \& Teacher Education International Conference. Vol. 2015. No. 1. 2015. 